Document downloaded from:

http://hdl.handle.net/10251/63159

This paper must be cited as:

Capella Hernández, JV.; Bonastre Pina, AM.; Ors Carot, R.; Peris Tortajada, M. (2015). An interference-tolerant nitrate smart sensor for Wireless Sensor Network applications. Sensors and Actuators B: Chemical. 213:534-540. doi:10.1016/j.snb.2015.02.125.

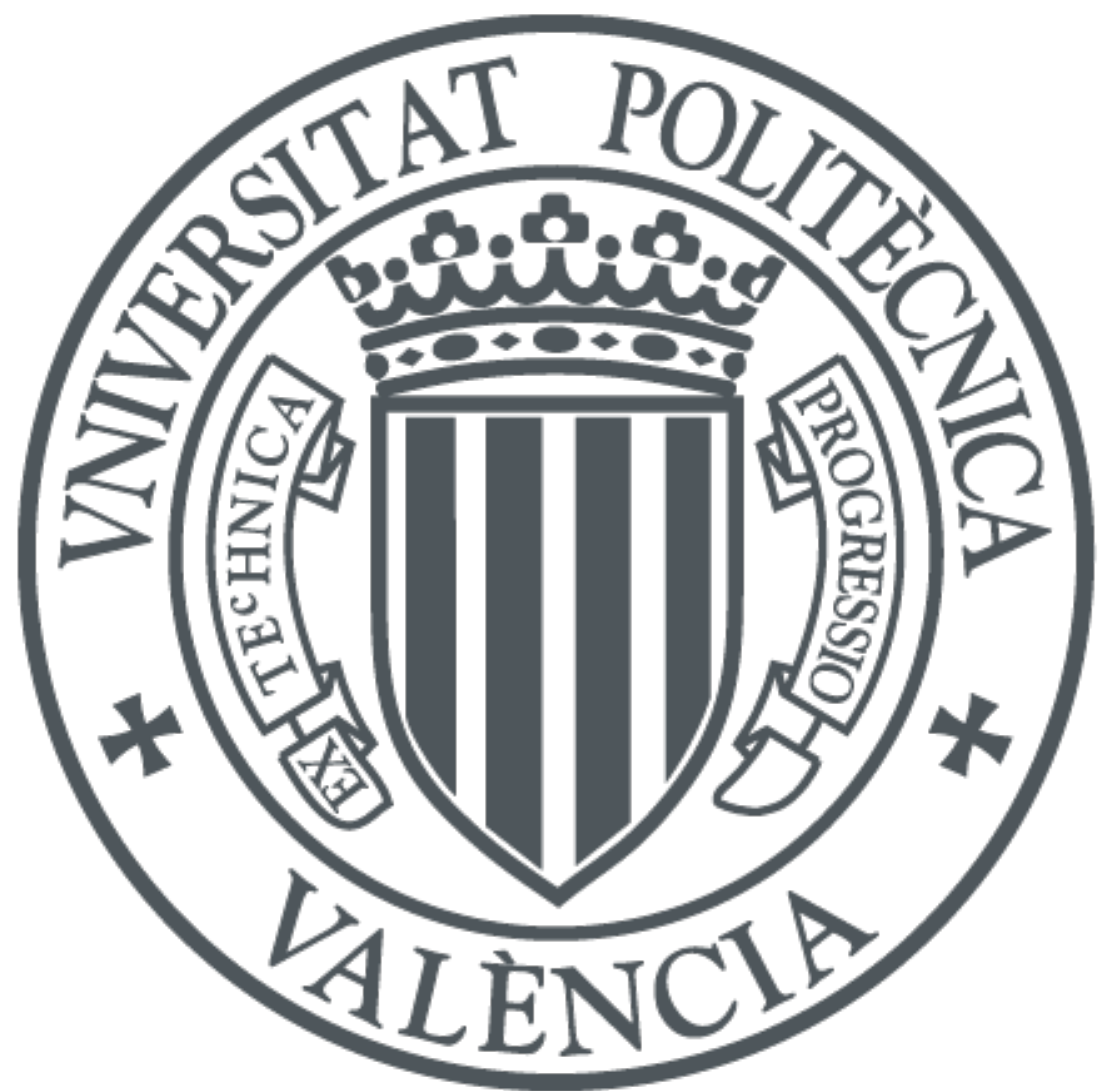

The final publication is available at

http://dx.doi.org/10.1016/j.snb.2015.02.125

Copyright Elsevier

Additional Information 


\section{Abstract}

\title{
An interference-tolerant nitrate smart sensor for Wireless Sensor Network applications
}

\author{
Juan V. Capella ${ }^{\text {a }}$, Alberto Bonastre ${ }^{\text {a }}$, Rafael Ors ${ }^{\text {a }}$, Miguel Peris ${ }^{b,{ }^{*}}$
}

${ }^{a}$ Department of Computer Engineering, Polytechnic University of Valencia, 46071 Valencia, SPAIN

${ }^{\mathrm{b}}$ Department of Chemistry, Polytechnic University of Valencia, 46071 Valencia, SPAIN

*Corresponding author; e-mail: mperist@qim.upv.es

As a major contaminant in ground water, nitrate determination is a common practice in environmental analysis, especially the continuous and simultaneous monitoring of its concentration at many different points. For this task, sensor networks are a promising tool, although they require the use of sensors with special features, such as those of Ion Selective Electrodes (ISEs). Unfortunately, their measurements are - to a greater or lesser extent - affected by the presence of other coexisting (interfering) ions. A new methodology is then proposed in this work to deal with major interferences (chloride and bicarbonate in the case of nitrate determination), in such a way that the results obtained in the measurements of the content of $\mathrm{NO}_{3}{ }^{-}$with a nitrate selective electrode can be considered as virtually error-free from these interferences. For this purpose, a new sensor node has been developed; it consists of three ISEs $\left(\mathrm{NO}_{3}^{-}, \mathrm{Cl}^{-}\right.$, and $\left.\mathrm{HCO}_{3}{ }^{-}\right)$ coupled to a low-consumption, low-cost microcontroller (a small chip containing all the computer components), which receives and processes all signals coming from the electrodes. This information is suitably treated, as described in detail in this paper, to provide an accurate estimation of the true value of $\mathrm{NO}_{3}{ }^{-}$concentration.

The application of this methodology results in an interference-tolerant nitrate smart sensor capable of being employed within a wireless sensor network in the continuous monitoring of nitrate concentration in aquifers and rivers.

Keywords: Smart sensor; Interference tolerance; Nitrate determination; Ion selective electrode; Interfering ion. 


\section{Introduction}

36 Nitrate is one of the most common contaminants of ground water, originating mainly

37 from agricultural fertilizer application and release of sewage. As the presence of this

38 species in water presents a well-known risk to health, it seems obvious that the

39 monitoring of nitrate concentration in aquifers and rivers may result fundamental.

40 Several analytical techniques have been used for this purpose [1], ion-selective

41 electrodes (ISEs) being perhaps the most suitable one; in this sense, the literature offers

42 a great deal of references, from early work [2] [3] to recent contributions especially with

43 a view of in-line monitoring [4] [5]. It is straightforward with advantages such as high

44 selectivity, sensitivity, good precision, simplicity, portability, non-destructive analysis,

45 and last but by no means least, low cost and power consumption. The latter makes this

46 technique highly suitable for Wireless Sensor Network (WSN) applications.

47 However, it is a common feature of all analysis methods that when atoms or ions of a

48 different species but with similar properties are also present, i.e. coexist with the atoms

49 or ions of interest, they interfere with measurement. This is also true of analysis using

50 ion selective electrodes, so that if ions similar to the target ions are present, they will -

51 to a greater or lesser extent - affect measurement (when considered in contrast to the

52 target ions, these ions are known as interfering ions). Therefore, when using the ion

53 electrode method, care needs to be taken with regard to mutual interference within each

54 of these groups [6].

55 In this sense, several attempts to deal with this problem have been carried out based on the joint consideration of several ISEs response. Some of these examples include the development of the so-called electronic tongues, arrays of potentiometric sensors (ISEs) coupled to pattern recognition tools. They have been applied to water quality monitoring [7][8], and their performances being compared to those of discrete conventional ion-selective electrodes [9]. On the other hand, recent efforts are focused on the development of artificial neural network (ANN) architectures; they have been applied to raw readings from a chemical sensor multi-probe (e-tongue), comprised of off-the shelf ISEs, to estimate individual ion concentrations in solutions at environmentally relevant concentrations and containing environmentally representative ion mixtures [10]. Nevertheless, all these approaches -though successful-are sometimes 
too complex (need of high-level computer resources) and time-consuming, and would not be adequate in case of WSN applications. That is why more simple developments, i.e. the utilization of discrete ISEs, are usually preferred.

No ion-selective electrodes are completely ion-specific [11]; all are sensitive to other ions having similar physical properties, to an extent which depends on the degree of similarity. Most of these interferences are weak enough to be ignored, but in some cases the electrode may actually be much more sensitive to the interfering ion than to the desired ion, requiring that the interfering ion be present only in relatively very low concentrations, or entirely absent. In practice, the relative sensitivities of each type of ion-specific electrode to various interfering ions is generally known and should be checked for each case; however the precise degree of interference depends on many factors, preventing precise correction of readings. Instead, the calculation of relative degree of interference from the concentration of interfering ions can only be used as a guide to determine whether the approximate extent of the interference will allow reliable measurements, or whether the experiment will need to be redesigned so as to reduce the effect of interfering ions.

The effects of coexisting ions can be predicted to some extent from the response membrane material, that is to say, the reactivity of the response membrane material to the coexisting ions. For example, a solid-state membrane electrode can be seriously affected by coexisting ions that form insoluble compounds or complex salts with the material of its response membrane; and a liquid membrane electrode can be affected by coexisting ions that form ionic associates with the components in its response membrane.

The intensity of the interference produced by an ion species is expressed by the selectivity coefficient (or by the maximum allowable coexistence factor, this roughly corresponding to the reciprocal of the selectivity coefficient). The Nicolsky-Eisenman equation (an extension to the Nernst equation) [12] defines the selectivity coefficient $k_{i j}$

$$
E_{i}=E_{i}^{0}+2.303 \frac{R T}{n_{i} F} \log \left\{a_{i}+\sum_{j} k_{i j}\left(a_{j}^{\left(n_{i} / n_{j}\right)}\right)\right\}
$$

where $E$ is the emf, $E^{0}$ the standard electrode potential, $n$ the ionic valency including the sign, $a$ the activity, $i$ the ion of interest (target ion), $j$ the interfering ions and $k_{\mathrm{ij}}$ is the selectivity coefficient. The smaller this value, the better the selectivity with respect to 
97 the target ion, i.e. the less the interference by $j[13]$.

98 Hence, in practice, the ratio of target ion to interfering ion concentration is very important. Higher concentrations of target ions result in the interfering ions having a smaller effect, and conversely, lower concentrations result in them having a larger effect. It is then logical that the ideal ISE should be interference-free, although up till now the objective of the major manufacturers of this type of devices consists of keeping these interferences to a minimum. Our aim is then to address cross-ion interferences in such a way that, although ISEs may be only partially selective for their target analyte, we can take advantage of their promising use in in-situ portable sensors.

Recent advances in the field of microelectronics and communications allow for the development of modern applications that require new sensors with different requirements from those of the traditional devices; additionally, and owing to their possibilities of data management, they permit to obtain more precise, robust, and powerful systems [14]. Following this line, in this paper we propose a nitrate smart sensor that is able to eliminate major interferences from other species, namely chloride and bicarbonate ions; moreover, it meets the necessary requirements to be utilized within an application based on WSN [15], with all its inherent benefits in the field of

114 chemical analyses.

115 In the next sections, the procedure proposed for the rejection of major interferences in

116 nitrate determination by ISEs will be described in detail, along with the results obtained

117 after its application to discrete samples, with a view to use it in the future within a WSN

118 for environmental analysis purposes.

\section{Rejection of interferences}

122 As mentioned above, one of the main drawbacks while measuring nitrate concentrations

123 by means of ISEs is the interference caused by other similar species, since their

124 presence may cause an incremental deviation on the results obtained. In case of the

125 nitrate electrode [16], the following ions usually interfere (average selectivity

126 coefficients, $\mathrm{SC}$, in brackets): Chloride $\left(6.10^{-3}\right)$, Bicarbonate $\left(5.10^{-3}\right)$, Nitrite $\left(1.10^{-3}\right)$,

127 Acetate $\left(5.10^{-4}\right)$, Fluoride $\left(1.10^{-4}\right)$, Sulfate $\left(1.10^{-5}\right)$. In this sense, the higher the value of

$128 \mathrm{SC}$, the more interference; therefore, in the present work we will consider those two 
129 interferences with the highest SC values, i.e. chloride and bicarbonate. Nevertheless, the

130 procedure could easily be applied to other - though less important - potential interfering

131 anions. It should also be remarked that, in our case, the chloride and bicarbonate

132 concentrations have been chosen according to the range found in previous field sample

133 analysis. On the other hand, in the field of Electroanalytical Chemistry it is generally

134 accepted that, at low ionic strengths, (e.g. below 0.01M for monovalent ions and

$1350.001 \mathrm{M}$ for divalent ions) the difference between concentration and activity is really

136 small and the use of concentration units instead of activity for the measurements

137 (including calibration) should not cause a significant error in the determinations, even

138 without the use of ionic strength adjustment buffer (ISAB). In our case, all occurring

139 ions being monovalent, the ionic strength of the highest concentrated solution is 0.005 ,

140 what means that concentrations will be utilized instead of activities with no significant

141 error.

142 The correction system is then based on the evaluation of the accumulative error suffered

143 by the obtained measurement. This error has been found to depend on three factors,

144 namely: the concentrations of nitrate, bicarbonate, and chloride. Our initial hypothesis

145 was that it is possible to estimate, and then compensate, the interference error if $\left[\mathrm{Cl}^{-}\right]$

146 and $\left[\mathrm{HCO}_{3}{ }^{-}\right]$are known. In this way, additional bicarbonate and chloride ISEs were

147 added to the nitrate ISE in order to measure all three concentrations. It must be

148 considered that the real concentrations are not available, as far as they have to be

149 determined by the measurements - probably affected by the interferences and perhaps

150 other instrumental errors - of these ISEs.

151 As described below, an exhaustive set of experiments (Table 2) has been carried out

152 under laboratory conditions. These experiments consisted of the application of the three

153 ISEs to mixtures prepared with known concentrations of all three anions. As expected,

154 measured nitrate concentrations suffered a relative error ranging from $15 \%$ to $30 \%$

155 (easily determined as the difference between measured values and true values). For their

156 part, the errors of both bicarbonate and chloride measurements were under $2 \%$ in all

157 instances.

158 In order to obtain reliable results, $n$ replications have been performed for each

159 measurement, $n$ being calculated as follows:

160 The results for each measurement have been considered as random variables $\left(\mathrm{X}_{1}\right.$,

$161 \mathrm{X}_{2}, \ldots, \mathrm{X}_{\mathrm{n}}$ ) with a $\mu$ mean value. $n$ simulations have been repeated until an estimation of

$162 \mu$ has been obtained with a $90 \%$ confidence interval according to the expression: 
$164 \bar{X}(n) \pm t_{n-1,0.95} \sqrt{S^{2}(n) / n}$

166 where $t_{n-1,0.95}$ represents the upper limit of the Student's t-distribution on $n$ - 1 degrees of

167 freedom, and $X(n)$ and $S^{2}(n)$ are the mean and the variance of the results obtained in the

168 different experiments. In general, 5-12 replications were carried out for each

169 measurement.

170 We have evaluated different techniques that relation the interferences of chloride and

171 bicarbonate on nitrate measurements, from simple, fixed (predetermined) corrections to

172 more complex techniques. From the corresponding essays, it could be noticed that the

173 former did not prove to be useful in all cases. More complex solutions yielded good

174 results, but their high requirements of computing resources make them unsuitable for

175 WSN. Instead, the proposed methodology brings together simplicity and highly

176 satisfactory results [17].

177 It assumes that there is some relationship between the concentration of interferents and

178 the error committed in the determination. Bearing this in mind, several experiments

179 were carried out using standard solutions; then, with the results obtained as well as the

180 corresponding true values, different mathematical procedures were evaluated to

181 determine the error committed. Least squares linear regression was chosen since it

182 provided negligible errors in the estimation. According to this procedure, the values

183 provided by the three ISEs used are plotted against (correlated with) the errors in nitrate

184 measurements (true values are known), the corresponding regression line coefficients

185 being shown in Table 1 . Linear regression has proved to be satisfactory $\left(\mathrm{R}^{2}=0.9607\right)$.

186 This accuracy is better, for example, than the measurement error.

187 This method also deals with cross-ion interferences, since the regression line is based on

188 measured ISE concentrations, rather than on true concentrations. Thus, the mutual

189 interferences have also been considered.

190 Thereafter, an estimation program was implemented; it applied the previously defined

191 error estimation procedure to the obtained measurements. The estimated error is then

192 subtracted to the nitrate concentration measured to obtain the corrected value. Once the

193 latter has been achieved, the error turns out to be under $5 \%$ in all studied cases. 


\section{System description}

197 The application of the technique described in the previous section results in the

198 development of a smart sensor node formed by three ISE-type transducers for the

199 determination of nitrate, chloride, and bicarbonate, respectively. The core of the system

200 consists of a low-consumption, low-cost microcontroller; its capacity is nevertheless

201 more than sufficient to carry out all the operations required to apply the developed

202 techniques. This microcontroller is a small integrated circuit that contains all the

203 computer components (CPU, memory, and necessary I/O subsystems), and therefore

204 offers the possibility to implement complete applications using only one chip [18]. The

205 device chosen (ARM Cortex M0) is a 32-bit microcontroller with a high energy

206 efficiency $(12.5 \mu \mathrm{W} / \mathrm{MHz})$ and performance; it has $2 \mathrm{~KB}$ RAM memory and $8 \mathrm{~KB}$

207 flash memory, as well as three timers (16 and 32 bits) and an A/D converter (10-bit

208 resolution and 8 channels). Incoming signals from ISEs are adapted/amplified by means

209 of an AD524 Instrumentation Amplifier (Analog Devices).

210 The capture of the information in the sensor node begins in the transducers (nitrate-ISE,

211 chloride-ISE, and bicarbonate-ISE). The analogical signals from these ISEs are

212 conveniently processed and amplified by means of analogical circuits, as shown in

213 Figure 1. The output of this circuit is received by the A/D converters of the

214 microcontroller (Input/Output subsystems) to be discretized. After the suitable

215 conversion, a digital information of each ISE measurement is available and, therefore,

216 able to be processed. From this moment, the error estimation procedure evaluates the

217 interference influence, and the measured nitrate concentration is then corrected by using

218 the proposed method, as described in Section 2.

219 Once the corrected measurement has been calculated, it is transmitted through the

220 communication subsystem, be it periodically, upon request, or in an automatic way

221 when certain conditions are met [19].

222 Since one of the goals was that this interference-tolerant nitrate smart sensor node could

223 be employed in future WSN applications (and thus within the future Internet of Things),

224 both its consumption and maintenance must reach a minimum value [20]. Therefore,

225 low consumption components (the ISEs and the microcontroller) have been utilized;

226 furthermore, the sensor node has been equipped with an energy harvesting subsystem

227 that is able to keep it on over long periods of time [2]. 


\section{4. Experimental}

230

231 All standard and reagent solutions were prepared from analytical reagent grade

232 chemicals $\left(\mathrm{KNO}_{3}\right.$ for nitrate, $\mathrm{NaHCO}_{3}$ for bicarbonate, and $\mathrm{KCl}$ for chloride) using

233 distilled and deionized water from a Milli-Q water purification system. The deionized

234 water had a specific conductivity less than $0.1 \mu \mathrm{s} \mathrm{cm}^{-1}$.

235 Nitrate, bicarbonate, and chloride concentrations are measured using three different

236 homemade ISEs. When the ISE is immersed in an aqueous solution, a potential is

237 established across the membrane that depends on the relative amounts of analyte in the

238 medium, this potential being read relative to a double-junction reference electrode of the

239 probe. Our ISEs were prepared and conditioned following some of the guidelines

240 provided by the literature [21].

241 In case of nitrate, the ISE employs a silver/silver chloride wire electrode in a custom

242 filling solution. The internal solution is separated from the sample medium by a

243 polyvinylchloride (PVC) membrane, which selectively interacts with $\mathrm{NO}_{3}{ }^{-}$ions. To

244 prepare this membrane, a mixture of $45 \mathrm{mg}$ tridodecylammonium nitrate, $370 \mathrm{mg}$

245 dibutylphthalate as plasticizer and $160 \mathrm{mg} \mathrm{PVC}$ is thoroughly dissolved in $7 \mathrm{~mL}$

246 tetrahydrofuran. The solution obtained is then poured into small glass rings and the

247 organic solvent is evaporated, thus achieving homogeneous membrane layers with a

248 thickness ranging between 450 and $500 \mu \mathrm{m}$. The resulting electrode has the following

249 characteristics: a Nernstian slope of $54 \pm 5 \mathrm{mV}$ per decade change in activity, a limit of

250 detection of $0.05 \mathrm{mg} \mathrm{L}^{-1}$ of $\mathrm{NO}_{3}^{-}$, a response time of less than 10 seconds, a selectivity

251 coefficient for nitrate against chloride $\left(k_{\mathrm{NO}^{-},}, \mathrm{Cl}^{-}\right)$of $5 \times 10^{-3}$, and a selectivity

252 coefficient for nitrate against bicarbonate $\left(k_{\mathrm{NO}_{3}-, \mathrm{HCO}^{-}}\right)$of $4 \times 10^{-3}$.

253 The nitrate-ISE was then calibrated with potassium nitrate standard solutions

254 maintained at $25^{\circ} \mathrm{C}$ and constantly stirred. Figure 2 shows a calibration curve for this

255 anion. All measurements (including calibration) were carried out with $100 \mathrm{~mL}$ of test

256 solution in a suitable Pyrex beaker. All glassware was decontaminated, washed several

257 times with double-distilled water and dried in an oven at $150^{\circ} \mathrm{C}$ overnight prior to use.

258 The validation of the results obtained by the nitrate-ISE was achieved using an official

259 standard method [22] and showed excellent correlation between the two techniques

$260\left(\mathrm{R}^{2}=0.9948\right)$ over a large range from 0.1 to $20 \mathrm{mg} \mathrm{L}^{-1}$ nitrate-N with no systematic

261 errors. On the other hand, the drift of the electrode contacting a $9.9 \mathrm{mM}$ nitrate solution 
262 is less than $0.4 \mathrm{mV} \mathrm{h}^{-1}$, measured at constant temperature and with ISE and reference

263 electrode continually immersed.

264 As regards bicarbonate, the ISE consists of a polyvinyl chloride tube covered with a thin

$265(10-25 \mu \mathrm{m}) \mathrm{HCO}_{3}{ }^{-}$-selective membrane made from a mixture containing polyvinyl

266 chloride, di-(2-ethylhexyl) sebacate, trioctyl tin chloride and an $\mathrm{H}^{+}$interference-

267 removing trifluroacetophenone (trifluoroacetyldecylbenzene), a liquid solution

268 containing $50 \mathrm{mM}$ phosphate buffer and $0.01 \mathrm{M}$ sodium chloride in the tube, and a lead

269 wire connected to a $\mathrm{Ag} / \mathrm{AgCl}$ reference electrode positioned in the tube. This ISE has

270 then the following features: a Nernstian slope of $55 \pm 5 \mathrm{mV}$ per decade change in

271 activity, a limit of detection of $1.5 \mathrm{mg} \mathrm{L}^{-1}$ of $\mathrm{HCO}_{3}^{-}$, and a response time of less than 20

272 seconds.

273 The bicarbonate-ISE was calibrated with sodium bicarbonate standard solutions

274 maintained at $25^{\circ} \mathrm{C}$ and constantly stirred. Figure 2 shows a calibration curve for this

275 anion. All measurements (including calibration) were carried out with $100 \mathrm{~mL}$ of test

276 solution in a suitable Pyrex beaker. All glassware was decontaminated, washed several

277 times with double-distilled water and dried in an oven at $150^{\circ} \mathrm{C}$ overnight prior to use.

278 The validation of the results obtained by the bicarbonate-ISE was performed using an

279 official standard method [22] and showed excellent correlation between the two

280 techniques $\left(\mathrm{R}^{2}=0.9936\right)$ over a large range from 10 to $250 \mathrm{mg} \mathrm{L}^{-1}$ bicarbonate with no

281 systematic errors. It should also be noted that the drift of the electrode contacting a 11.9

$282 \mathrm{mM}$ bicarbonate solution is less than $0.5 \mathrm{mV} \mathrm{h}^{-1}$, measured at constant temperature and

283 with ISE and reference electrode continually immersed.

284 Finally, in the case of the chloride-ISE, the membrane is made from a combination of

285 ([ $\gamma$-[4,5-dimethyl-3,6-bis(octyloxy)-1,2-phenylene]]bis(trifluoroacetato-O)dimercury),

286 tridodecylmethylammonium chloride, bis(2-ethylhexyl)sebacate, and polyvinylchloride,

287 a reference solution containing $0.1 \mathrm{M}$ potassium chloride, and a wire connected to a

$288 \mathrm{Ag} / \mathrm{AgCl}$ reference electrode. The ISE so constituted is characterized by a Nernstian

289 slope of $54 \pm 5 \mathrm{mV}$ per decade change in activity, a limit of detection of $1 \mathrm{mg} \mathrm{L}^{-1} \mathrm{of} \mathrm{Cl}^{-}$,

290 and a response time of less than 10 seconds.

291 The chloride-ISE was calibrated with potassium chloride standard solutions kept at

$29225^{\circ} \mathrm{C}$ and constantly stirred. Figure 2 shows a calibration curve for this anion. All

293 measurements (including calibration) were carried out with $100 \mathrm{~mL}$ of test solution in a

294 suitable Pyrex beaker. All glassware was decontaminated, washed several times with

295 double-distilled water and dried in an oven at $150^{\circ} \mathrm{C}$ overnight prior to use. 
296 The validation of the results obtained by the chloride-ISE was performed using an

297 official standard method [22] and showed excellent correlation between the two

298 techniques $\left(\mathrm{R}^{2}=0.9963\right)$ over an approximate range between 1 and $300 \mathrm{mg} \mathrm{L}^{-1}$ chloride

299 with no systematic errors. And last but not least, the drift of the electrode contacting a

$300 \quad 13.4 \mathrm{mM}$ chloride solution is less than $0.4 \mathrm{mV} \mathrm{h}^{-1}$, measured at constant temperature and

301 with ISE and reference electrode continually immersed.

302 Stock solutions of $\mathrm{KNO}_{3}$ (for nitrate), $\mathrm{NaHCO}_{3}$ (for bicarbonate), and $\mathrm{KCl}$ (for

303 chloride) were prepared by dissolving the appropriate amount of reagent in distilled-

304 deionized water. In order to carry out the measurements, the three aforementioned ISEs

305 were then simultaneously immersed in solutions obtained by suitable mixtures of the

306 stock solutions, in such a way that the following concentrations were selected: (a) 0.0 ,

$3070.5,1.0,1,5$, and $2.0 \mathrm{mg} \mathrm{L}^{-1}$ of nitrate, (b) $0,125,150,175$, and $200 \mathrm{mg} \mathrm{L}^{-1}$ of

308 bicarbonate, and (c) $0,50,100,150$, and $200 \mathrm{mg} \mathrm{L}^{-1}$ of chloride. The contents of the

309 three analytes were then measured in combinations of these solutions in threes (Table

3102 2).

311

\section{Results and discussion}

314 The results achieved are summarized in Figures 3 and 4. Figure 3 shows the values

315 obtained without applying the proposed methodology. The horizontal axis represents the

316 true values of $\left[\mathrm{NO}_{3}{ }^{-}\right]$(solutions were prepared by us following the procedure described

317 in the previous section, and are therefore known values), whereas the vertical axis

318 represents the measured concentrations, i.e. the experimental values provided by the

319 ISEs. Needless to say that an ideal nitrate sensor should give rise to a 45-degree line to

320 which the red line of the figure approximates a lot. This line reflects the results obtained

321 with the nitrate-ISE after its calibration and in the absence of interferences (i.e.

322 immersed in a standard nitrate solution). On the other hand, when the interfering anions

323 (chloride and bicarbonate) are added at known concentrations, the measured values start

324 to distance themselves from real concentrations owing to the interferences.

325 Consequently, an uncertainty area appears depending on the concentration of the

326 interfering species. For the concentration ranges of the interferents (see section 4), this

327 uncertainty area is the one between the upper and lower lines.

328 As it can be inferred from our study, and on the basis of a known nitrate concentration 
329 (i.e. any point at the horizontal axis), the response of nitrate-ISE may vary - as a

330 function of the concentration of interferents - in the range resulting from the

331 intersection of the vertical line at this point with the uncertainty area. As an example, for

332 a known value of $\left[\mathrm{NO}_{3}^{-}\right]=2 \mathrm{mg} \mathrm{L}^{-1}$ the results that could be obtained by measuring

333 with the corresponding ISE would lie between $2.04 \mathrm{mg} \mathrm{L}^{-1}$ (no interferences) and 2.52

$334 \mathrm{mg} \mathrm{L}^{-1}$ (corresponding to $\left[\mathrm{Cl}^{-}\right]=200 \mathrm{mg} \mathrm{L}^{-1}$ and $\left[\mathrm{HCO}_{3}^{-}\right]=200 \mathrm{mg} \mathrm{L}^{-1}$ ). It can be

335 noticed that - depending on the concentrations of interferents - the error obtained can

336 be significant, up to $26 \%$.

337 On the other hand, attention should also be paid to the real case of the need to determine

338 (using $\mathrm{a} \mathrm{NO}_{3}^{-}$-ISE) the concentration of nitrate in the presence of an unknown amount

339 of chloride and bicarbonate. In these circumstances, the starting point is the vertical axis

340 (measured values of nitrate-ISE); true nitrate concentrations in the sample may be

341 anyone corresponding to the intersection of a horizontal line passing at this point with

342 the uncertainty area. For instance, for a measured concentration of $2 \mathrm{mg} \mathrm{L}^{-1}$, true values

343 of $\left[\mathrm{NO}_{3}^{-}\right]$in the analyzed sample may be in the range between $1.56 \mathrm{mg} \mathrm{L}^{-1}$ (highest

344 concentration of interferents) and $1.96 \mathrm{mg} \mathrm{L}^{-1}$ (no interferences). The measurement

345 uncertainty is again really high.

346 Figure 4 shows the results obtained after the data treatment of previous measurements

347 with the method described in section 2 . The values yielded by a node using this

348 procedure would also generate (as described above for Figure 3) an uncertainty area,

349 although in this case it is significantly smaller. This fact makes the measurements from

350 this node to be virtually free from the effects of the interferences, unlike in case this

351 methodology would not be used.

352 Indeed, if a given value of $\left[\mathrm{NO}_{3}{ }^{-}\right]$is now considered and a vertical line is drawn through

353 this point, its intersection with the uncertainty area yields a much lower dispersion of

354 values. In case of the above mentioned example $\left(\left[\mathrm{NO}_{3}{ }^{-}\right]=2.00 \mathrm{mg} \mathrm{L}^{-1}\right)$, the values

355 obtained vary between $1.99 \mathrm{mg} \mathrm{L}^{-1}$ (for $100 \mathrm{mg} \mathrm{L}^{-1}$ chloride and $125 \mathrm{mg} \mathrm{L}^{-1}$

356 bicarbonate) and $2.07 \mathrm{mg} \mathrm{L}^{-1}$ (for $150 \mathrm{mg} \mathrm{L}^{-1}$ chloride and $200 \mathrm{mg} \mathrm{L}^{-1}$ bicarbonate), that

357 is to say, with a maximum error of $3.5 \%$, far from the aforementioned $26 \%$.

358 Again, we return to the real case of the need to determine (using a $\mathrm{NO}_{3}^{-}$-ISE) the

359 concentration of nitrate in the presence of an unknown amount of chloride and

360 bicarbonate. A point is taken at the vertical axis (the value corresponding to the

361 measurement obtained using the proposed methodology), for instance $2.00 \mathrm{mg} \mathrm{L}^{-1}$

362 nitrate; when a horizontal line is drawn through this point, it intersects with the 
363 uncertainty area at values ranging from $1.93 \mathrm{mg} \mathrm{L}^{-1}$ (for $200 \mathrm{mg} \mathrm{L}^{-1}$ chloride and 175

$364 \mathrm{mg} \mathrm{L}^{-1}$ bicarbonate) to $1.99 \mathrm{mg} \mathrm{L}^{-1}$ (for $50 \mathrm{mg} \mathrm{L}^{-1}$ chloride and $125 \mathrm{mg} \mathrm{L}^{-1}$ bicarbonate).

365 This demonstrates that the resulting nitrate concentration is very close to the true value,

366 the error being thus considerably reduced.

\section{Conclusions}

This work presents a new methodology to develop smart sensors with interference tolerance based on simple Ion Selective Electrodes (ISE). For this purpose, a correction mechanism based on interferent concentration measurement, error estimation, and a correction procedure has been added. This method permits to "reject" the mutual contributions to the analytical signals of ISEs due to the presence of interfering species in the analyzed samples. Basically, it consists of applying statistical techniques -linear regression of experimental data- to adjust the linear coefficients of each interferent on the basis of the experiments observed.

378 This has been incorporated into the design and implementation of a nitrate smart sensor for WSN applications that is able to overcome the problem associated with interfering anions in ground waters, namely, chloride and bicarbonate. The procedure has been applied to the measurements obtained from a nitrate-ISE in the presence of those two anions by means of a microcontroller. The smart sensor node is then composed of the three ISEs used $\left(\mathrm{NO}_{3}^{-}, \mathrm{Cl}^{-}, \mathrm{HCO}_{3}^{-}\right)$along with a low-cost, low-consumption microcontroller. All three ISE's are simultaneously immersed in a water sample; the corresponding measurements obtained are then conveniently received and processed by the microcontroller. The final result after applying the proposed method is that the nitrate measurements performed by this smart sensor turn out to be virtually interference-free.

389 Finally, the significance of this proposal lies in the application of automatic procedures

390 implemented in microcontrollers in order to compensate physical deficiencies, such as

391 the impact of interferences, in measurement processes. This proposal may be extended

392 to increase the smartness of sensor nodes in order to enhance their features.

393 New-generation smart sensors applying these principles may be suitable for integration

394 in Wireless Sensor Networks, on which Internet of Things and Ambient Intelligence will 395 be based. 


\section{Acknowledgements}

398

399 The authors gratefully acknowledge the financial support from the Valencian Regional

400 Government under Research Project GV/2014/012, Polytechnic University of Valencia

401 (Research Project UPV PAID-06-12) and Spanish Government (Research Projects

402 CTM2011-29691-C02-01 and TIN2011-28435-C03-0).

403

\section{References}

405

406 [1] L.M. Nollet (Ed.), Handbook of water analysis, CRC Press, Boca Raton, FL (USA), 4072007.

408 [2] G.J. Moody, J.D.R. Thomas, Selective Ion-sensitive Electrodes, Sel. Annu. Rev.

409 Anal. 3 (1973) 59-138.

410 [3] W. Simon, E. Pretsch, D. Ammann, W.E. Morf, M. Güggi, R. Bissig, M. Kessler,

411 Recent developments in the field of ion selective electrodes, Pure Appl. Chem. 42 412 (1975) 613-626.

413 [4] J.V. Capella, A. Bonastre, R. Ors, M. Peris, In line river monitoring of nitrate

414 concentration by means of a Wireless Sensor Network with energy harvesting, Sens.

415 Actuators B: Chem. 177 (2013) 419-427.

416 [5] J.V. Capella, A. Bonastre, R. Ors, M. Peris, A step forward in the in-line river

417 monitoring of nitrate by means of a wireless sensor network, Sens. Actuators B: Chem. 418195 (2014) 396-403.

419 [6] G. Dimeski, T. Badrick, A.S. John, Ion Selective Electrodes (ISEs) and interferences 420 - A review, Clin. Chimica Acta 411 (2009) 309-317.

421 [7] A.V. Legin, E.A. Bychkov, B.L. Seleznev, Y.G. Vlasov, Development and analytical 422 evaluation of a multisensor system for water quality monitoring, Sens. Actuators B:

423 Chem. 27 (1995) 377-379.

424 [8] R. Martínez-Máñez, J. Soto, E. García-Breijo, L. Gil, J. Ibáñez, E. Llobet, An

425 "electronic tongue" design for the qualitative analysis of natural waters, Sens. Actuators 426 B: Chem. 104 (2005) 302-307. 
427 [9] A.V. Legin, A.M. Rudnitskaya, Y.G. Vlasov, C. di Natale, A. D’Amico, The features 428 of the electronic tongue in comparison with the characteristics of the discrete ion429 selective sensors, Sens. Actuators B: Chem. 58 (1999) 464-468.

430 [10] A.V. Mueller, H.F. Hemond, Extended artificial neural networks: Incorporation of $a$ 431 priori chemical knowledge enables use of ion selective electrodes for in-situ 432 measurement of ions at environmentally relevant levels, Talanta, 117 (2013) 112-118.

433 [11] P. Bühlmann, E. Pretsch, E. Bakker, Carrier-Based Ion-Selective Electrodes and 434 Bulk Optodes. 2. Ionophores for Potentiometric and Optical Sensors, Chem. Rev. 98 435 (1998) 1593-1687.

436 [12] C.G. Zoski (Ed.), Handbook of Electrochemistry, Elsevier, Amsterdam, 2007.

437 [13] Y. Umezawa, P. Bühlmann, K. Umezawa, K. Tohda, S. Amemiya, Potentiometric 438 selectivity coefficients of ion-selective electrodes, Pure and Applied Chemistry, 72 439 (2000) 1851-2082.

440 [14] S. Oteafy, S.H. Hassanein, Current hindrances in WSNs, John Wiley \& Sons, New 441 York, NY (USA) 2014.

442 [15] J. Suhonen, M. Kohvakka, V. Kaseva, T.D. Hämäläinen, M. Hännikäinen, Low443 Power Wireless Sensor Networks, Springer, New York, NY (USA), 2012.

444 [16] M.J. Moorkroft, J. Davis, R.G. Compton, Detection and determination of nitrate: a 445 review, 54 (2001) 785-803.

446 [17] Y. Liu, B.C. Seet, A. Al-Anbuky, Ambient Intelligence Context-Based Cross447 Layer Design in Wireless Sensor Networks, Sensors 14 (2014) 19057-19085.

448 [18] O. Casas, M. López, M. Quílez, X. Martinez-Farre, G. Hornero, C. Rovira, M.R. 449 Pinilla, P.M. Ramos, B. Borges, H. Marques, P. Girão, Wireless sensor network for 450 smart composting monitoring and control, Measurement 47 (2014) 483-495.

451 [19] J.V. Capella, A. Bonastre, R. Ors, M. Peris, A wireless sensor network approach for 452 distributed in-line chemical analysis of water, Talanta 80 (2010) 1789-1798.

453 [20] N. Zaman, K. Ragab, A.B. Abdullah, Wireless Sensor Networks and Energy 454 Efficiency: Protocols, Routing and Management, IGI Global, Hershey, PA (USA) 2012. 455 [21] E. Pretsch, The new wave of ion-selective electrodes, TrAC Trends Anal. Chem. $45626(2007) 46-51$.

457 [22] G.W. Latimer (Ed.), AOAC International, Official Methods of Analysis of AOAC 458 International, $19^{\text {th }}$ ed., AOAC, Washington D.C. 2012. 


\section{$461 \quad$ Legends of figures}

462

463 Figure 1.- Structure of a nitrate smart sensor node. A = signal amplifier \& conditioner.

464 Figure 2.- Calibration plots for the determination of nitrate, bicarbonate, and chloride using the corresponding ion-selective electrodes. Activities are expressed in mol L-1.

466 Figure 3.- Experimental results obtained in the presence of interferences. Values between parenthesis on the right side of the figure denote the following: $\left(\left[\mathrm{HCO}_{3}{ }^{-}\right],\left[\mathrm{Cl}^{-}\right]\right) \mathrm{All}$

Figure 4.- Experimental results obtained with interference tolerance. Values between parenthesis on the right side of the figure denote the following: $\left(\left[\mathrm{HCO}_{3}{ }^{-}\right],\left[\mathrm{Cl}^{-}\right]\right)$All concentrations are expressed in $\mathrm{mg} \mathrm{L}^{-1}$. 
473 Table 1.- Coefficients obtained in the regression analysis (concentrations in $\mathrm{mg} \mathrm{L}^{-1}$ )

474

475

476

477

478

479

480

481

482

483

\begin{tabular}{lc}
\multicolumn{1}{c}{ Coefficient } & \multicolumn{1}{c}{ Value } \\
Intercept & \\
{$\left[\mathrm{NO}_{3}^{-}\right]$} & $-0,108336504$ \\
{$\left[\mathrm{Cl}^{-}\right]$} & 0,123178928 \\
{$\left[\mathrm{HCO}_{3}{ }^{-}\right]$} & 0,000920765 \\
& 0,000386705
\end{tabular}


484 Table 2.- Initial concentration data set

485

\begin{tabular}{|c|c|c|c|}
\hline$\left[\mathrm{NO}_{3}{ }^{-}\right], \mathrm{mg} \mathrm{L}^{-1}$ & {$\left[\mathrm{Cl}^{-}\right], \mathrm{mg} \mathrm{L}^{-1}$} & {$\left[\mathrm{HCO}_{3}{ }^{-}\right], \mathrm{mg} \mathrm{L}^{-1}$} & TOTAL \\
\hline 0.5 & 125 & 50 & \\
\hline 1.0 & 150 & 100 & 64 experiments \\
\hline 1.5 & 175 & 150 & 04 expermments \\
\hline 2.0 & 200 & 50 & \\
\hline
\end{tabular}

486 